\title{
Congenital H-type tracheo-oesophageal fistula
}

\author{
B. SUNDAR, E. J. GUINEY, and BARRY O'DONNELL \\ From Our Lady's Hospital for Sick Children, Crumlin, Dublin, Eire
}

\begin{abstract}
Sundar, B., Guiney, E. J., and O'Donnell, B. (1975). Archives of Disease in Childhood, 50, 862. Congenital H-type tracheo-oesophageal fistula. Five cases of congenital H-type tracheo-oesophageal fistula are presented. The clinical features common to all were choking and coughing precipitated by feeds, recurrent chest infections, and episodes of abdominal distension. The usefulness of repeated oesophagograms in diagnosing the condition is emphasized. Operation in all cases was by the cervical approach. The single postoperative death was related to pre-existent severe chest infection, and no postoperative complications were encountered in the other patients.
\end{abstract}

Despite the elapse of a century since the original description of this condition by Lamb (1873) it continues to be often unrecognized. We report here 5 cases seen in Our Lady's Hospital for Sick Children and St. Joseph's Hospital, Dublin, between 1971 and 1974.

\section{Case reports}

Case 1. A girl who had been investigated in two hospitals for recurrent attacks of chest infection and episodes of abdominal distension dating back to birth. Barium swallow examinations at 10 and 15 months were reported as normal. Finally, an oesophagogram, with the dye injected through a catheter placed in the oesophagus, showed tracheal spill through a fistula at the level of the second and third dorsal vertebrae. She was operated on when $2 \frac{1}{2}$ years of age through a left supraclavicular approach. Uneventful recovery followed.

Case 2. A 7-day-old female infant was admitted with a history of being 'mucousy' since birth. This was associated with abdominal distension. The child became cyanosed after admission and chest $x$-ray examination showed right upper zone pneumonitis. Barium swallow examination 8 days later did not show anything abnormal, but a repeat examination outlined a fistula. The fistula was divided through a left supraclavicular approach. The infant died 10 hours after the operation. Post-mortem examination showed extensive bronchopneumonia.

Case 3. A female newborn infant was admitted with a history of respiratory distress, abdominal distension, and vomiting. The child developed right-sided pneumonitis within the first 7 days. Barium enema and

Received 26 February 1975. meal examinations were normal apart from revealing uncomplicated malrotation. Gastrografin swallow examination at one month of age showed evidence of a vascular ring. At 37 days of age an aberrant right subclavian artery was divided through a left thoracotomy. The child failed to improve after this and weighed only $6.12 \mathrm{~kg}$ when 10 months old. A repeat oesophagogram then revealed a fistula. Division of this through a left cervical approach was followed by uneventful recovery.

Case 4. A female infant was admitted when 2 months old with a history that she became distressed after every feed with vomiting, cyanosis, and dyspnoea and also had episodes of abdominal distension. Chest $x$-rays showed patchy pneumonitis on the right side. An oesophagogram showed an $\mathrm{H}$-fistula at the level of the first thoracic vertebra. The child was operated upon at 10 weeks of age through a left cervical approach and made an uneventful recovery.

Case 5. A male infant was detained in a neonatal unit for 5 weeks after birth because of a chest infection, and was readmitted when 3 months old for a recurrence of the same complaint. When he was admitted to our hospital at 9 months of age for a chest infection an oesophagogram showed an H-type fistula. This was divided through a left supraclavicular approach. Postoperative recovery was uneventful.

\section{Discussion}

Tracheo-oesophageal fistula without atresia has always been assumed to be a rare condition. The reported incidence varies from $1.8 \%$ (Waterston, Carter, and Aberdeen, 1962) to $4 \cdot 2 \%$ (Holder et al., 1964) among all types of tracheo-oesophageal fistula. During the 4-year period under review the 5 cases reported above constituted $8 \cdot 3 \%$ of all 
types, which is a higher figure than found in published reports. The high incidence in our series may be chance. However, we in the Republic of Ireland are particularly well situated to estimate accurately the incidence of congenital abnormalities among our newborn. The country is small and confined, emigration has virtually ceased, and personal communication is frequent between the doctors in charge of maternity and baby units.

The triad of choking and coughing precipitated by feeds, recurrent chest infections, and episodes of abdominal distension was seen repeatedly in these 5 cases, though the emphasis tended to vary greatly. For example, abdominal distension in Case 2 was so marked as to suggest a diagnosis of Hirschsprung's disease. In a large survey of reported cases Killen and Greenlee (1965) noted that the diagnosis was made within the first month of life in $43 \%$ and within the first year in $83 \%$. In the present series the ear.iest diagnosis was made at 2 months of age (Cases 2 and 4 ) and 4 out of the 5 were diagnosed within the first year of life. Nevertheless, the symptoms dated back to birth in all the 5 cases and therefore the chance of an earlier diagnosis existed.

We did not find bronchoscopy or oesophagoscopy helpful, perhaps because skilled radiological demonstration of the fistula in each case made endoscopy unnecessary. Hays, Woolley, and Snyder (1966) found oesophagoscopy unhelpful. Bedard, Girvan, and Shandling (1974) showed the fistula at bronchoscopy in 12 out of 15 patients. They found oesophagoscopy alone to be useless. Probably the modern, fine infant endoscopes will be more useful. We did not have to do an exploratory operation to establish the diagnosis in any of our cases, as suggested by Moncrief and Randolph
(1966), but the importance of often-repeated oesophagograms in avoiding delay in diagnosis must be emphasized.

The fistula was successfully divided in our 5 cases by a cervical approach on the left side. This has also been the experience of others (Schneider and Becker, 1962; Lynn and Davies, 1961; Moncrief and Randolph, 1966). The single postoperative death in our series was due to bronchopneumonia related to pre-existing chest infection-a constant feature of this syndrome. There were no postoperative complications such as recurrent fistula or recurrent laryngeal nerve damage in the remaining 4 cases.

\section{REFERENCES}

Bedard, P., Girvan, D. P., and Shandling, B. (1974). Congenital H-type tracheoesophageal fistula. Fournal of Pediatric Surgery, 9, 663 .

Hays, D. M., Woolley, M. M., and Snyder, W. H. (1966). Esophageal atresia and tracheoesophageal fistula. Management of the uncommon types. Fournal of Pediatric Surgery, 1, 240.

Holder, T., Cloud, D. T., Lewis, J. B., and Pilling, G. P. (1964). Esophageal atresia and tracheoesophageal fistula. Pediatrics, 34, 542.

Killen, D., and Greenlee, H. B. (1965). Transcervical repair of H-type congenital tracheoesophageal fistula. Annals of Surgery, $162,145$.

Lamb, D. S. (1873). A fatal case of congenital tracheoesophageal fistula. Philadelphia Medical Times, 3, 705.

Lynn, H. B., and Davis, L. A. (1961). Tracheoesophageal fistula without atresia of the esophagus. Surgical Clinics of North America, 41, 871.

Moncrief, J. A., and Randolph, J. G. (1966). Congenital tracheoesophageal fistula without atresia of the esophagus: method for diagnosis and surgical correcion. Fournal of Thoracic and Cardiovascular Surgery, 51, 434.

Schneider, K. M., and Becker, J. M. (1962). The 'H-type' tracheoesophageal fistula in infants and children. Surgery, 51, 677.

Waterston, D. J., Carter, R. E. B., and Aberdeen, E. (1962). Esophageal atresia: tracheoesophageal fistula. Lancet, 1, 819.

Correspondence to Dr. B. Sundar, Columbus Hospital, 2520 North Lakeview Avenue, Chicago, Ill., 60614 USA. 